\title{
Multiples Myelom als seltene Ursache eines Pancoast-Tumors
}

\author{
Multiple Myeloma as a Rare Cause of a Pancoast Tumor
}

Autoren

Institute
S. Kweider ${ }^{1}$, D. Frechen ${ }^{1}$, C. Hausdorf ${ }^{1}$, A. Heinzel ${ }^{2}$, S. Krüger ${ }^{1}$

${ }^{1}$ Medizinische Klinik I, Universitätsklinikum RWTH Aachen

${ }^{2}$ Klinik für Nuklearmedizin, Universitätsklinikum RWTH Aachen $\begin{array}{ll}\text { eingereicht } 22.8 .2012 & 23.8012\end{array}$

akzeptiert 23.8.2012

Bibliografie

DOI http://dx.doi.org/

$10.1055 / \mathrm{s}-0032-1325687$

Online-Publikation: 25.9.2012

Pneumologie 2012; 66: 666-668

(c) Georg Thieme Verlag KG

Stuttgart · New York

ISSN 0934-8387

Korrespondenzadresse Prof. Dr. med. Stefan Krüger

Medizinische Klinik I

Universitätsklinikum Aachen

Pauwelsstr. 30

52074 Aachen

stkrueger@ukaachen.de
Eine 60-jährige Patientin stellte sich in unserer Ambulanz zur Abklärung eines bei einer ambulanten Untersuchung radiologisch aufgefallenen großen Pancoast-Tumors der rechten Lunge vor ( $\bullet$ Abb. 1). Das Röntgen-Thoraxbild war aufgrund seit vier Monaten bestehender Schmerzen im rechten Arm und der rechten Schulter durchgeführt worden. Bis auf eine Nikotinanamnese mit ca. 5 pack years bestanden keine relevanten Vorerkrankungen.

In der schon extern vorab durchgeführten CTThorax-Untersuchung ( Abb.2) fanden sich eine Osteolyse in der rechten Scapula sowie die $6 \times 4 \times 4,7 \mathrm{~cm}$ große Raumforderung im rechten Lungenapex mit Infiltration der oberen Thoraxapertur bis in den 2. Brustwirbelkörper hinein mit Einengung des Spinalkanals.

Bronchoskopisch zeigte sich ein unauffälliger Befund, die transbronchiale Biopsie der Raumforderung war nicht diagnostisch. Die histologische Sicherung erfolgte daher mittels CT-gesteuerter Punktion. Überraschenderweise ergab sich immunhistologisch kein Hinweis auf das klinisch vermutete Bronchialkarzinom, sondern der Nachweis eines Multiplen Myeloms. Die Gammopathiediagnostik im Urin (Immunfixation) ergab bei geringer Proteinurie $(259 \mathrm{mg} / \mathrm{l})$ den Nachweis von monoklonalen Lambda-Leichtketten im Sinne einer Bence-Jonce-Proteinurie. Die SerumElektrophorese zeigte keinen Hinweis auf eine Paraproteinämie. Eine Knochenmarkpunktion konnte keinen Knochenmarksbefall durch das multiple Myelom nachweisen. Ein ergänzendes MRT des Tumors inkl. des BWK 2 konnte noch klarer die tumoröse Infiltration der Thoraxwand mit Destruktion der 1. Rippe und dem Wachstum per continuitatem in den HWK 7 und BWK 1 und BWK 2 rechtsseitig mit Tumoreinbruch in den Spinalkanal bestätigen ( $\bullet$ Abb. 3 und $\bullet$ Abb.4). Im PET-CT $($ Abb.5) war schließlich doch ein polytoper ossärer Befall mit Nachweis von Läsionen in der rechten Spina iliaca anterior, im rechtem

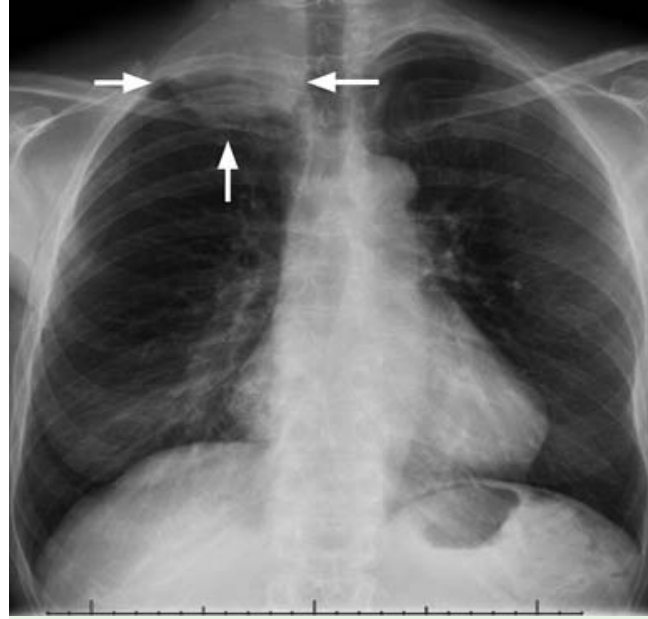

Abb. 1 Röntgen-Thorax p.a. bei Aufnahme. Große oval konfigurierte Verdichtung (Pfeile) in Projektion auf den rechten Lungenapex.

Os ilium sowie im rechten Femurhals und -schaft sichtbar:

Als abschließende Diagnose des Pancoast-Tumors rechts handelte es sich somit um ein Multiples Myelom. Es wurde eine Radiatio der Osteolyse in der rechten Schulter und anschließend eine systemische Chemotherapie mit Velcade und Dexamethason eingeleitet.

Pancoast-Tumore, auch „Sulcus-superior-Tumore“ genannt, finden sich im Lungenapex und machen ca. 5\% der Bronchialkarzinome aus. Histologisch handelt es sich am häufigsten um Plattenepitheloder Adenokarzinome. Aufgrund ihrer Lage infiltrieren sie früh die Brustwand, Rippen, Wirbelsäule, Plexus brachialis und benachbarte Gefäße. Wie auch bei unserer Patientin besteht die charakteristische Symptomatik aus Schmerzen in der Schulter, Arm und Hand (Plexusneuralgie). Dies wird nicht selten als orthopädische Ursache an Halswirbelsäule, Schulter oder Ellbogen verkannt. In ca. 20\% der Fälle kann es durch eine Sympathikusläsion zu einem Horner-Syndrom 


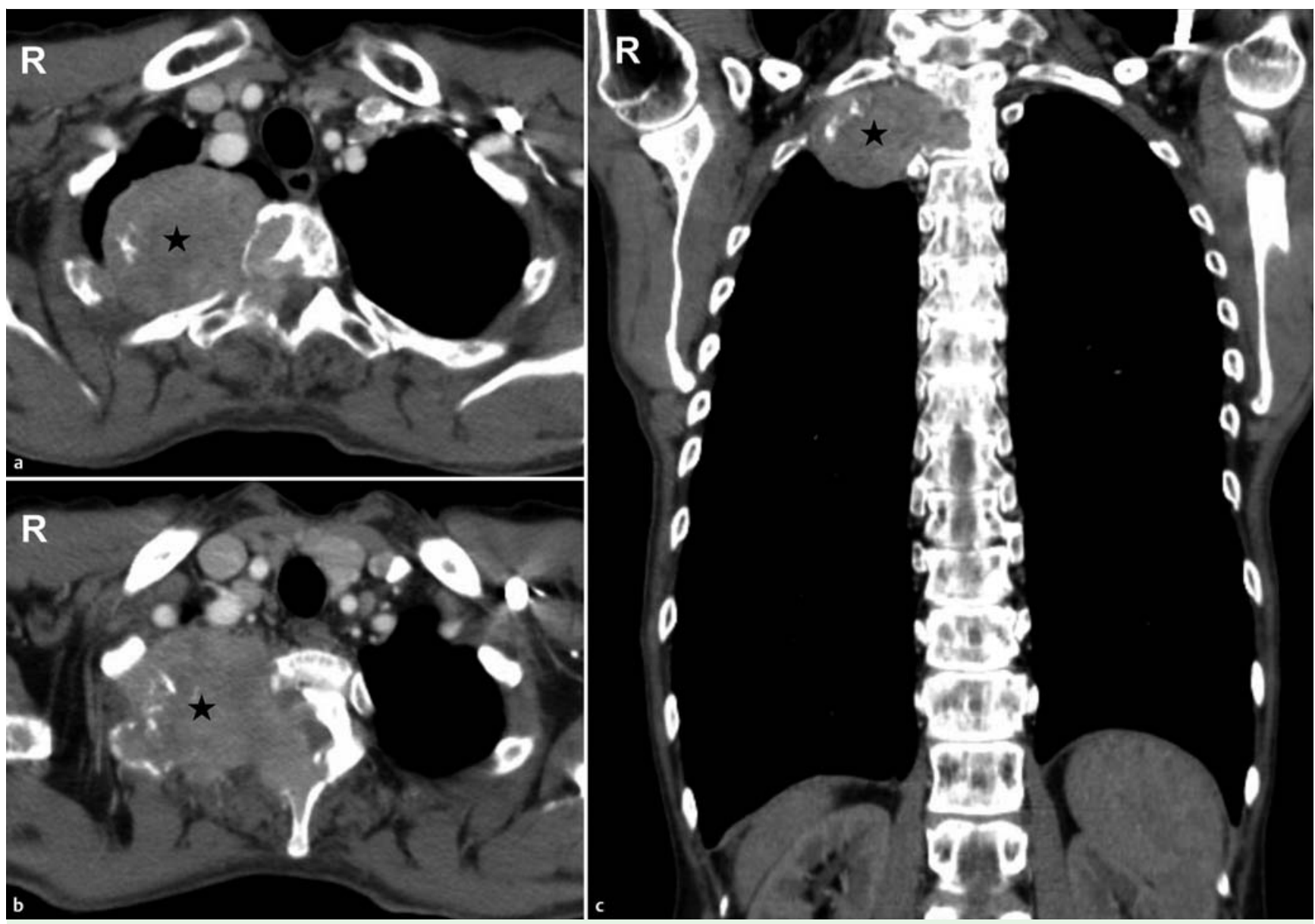

Abb.2 Computertomografie Thorax. In den transversalen Schnitten Darstellung des großen Pancoast-Tumors (*) mit Infiltration der Umgebung bis in den 2. Brustwirbelkörper (a und b). In der koronaren Ebene (c) sichtbare ossäre Destruktion von HWK 7 und BWK 1.
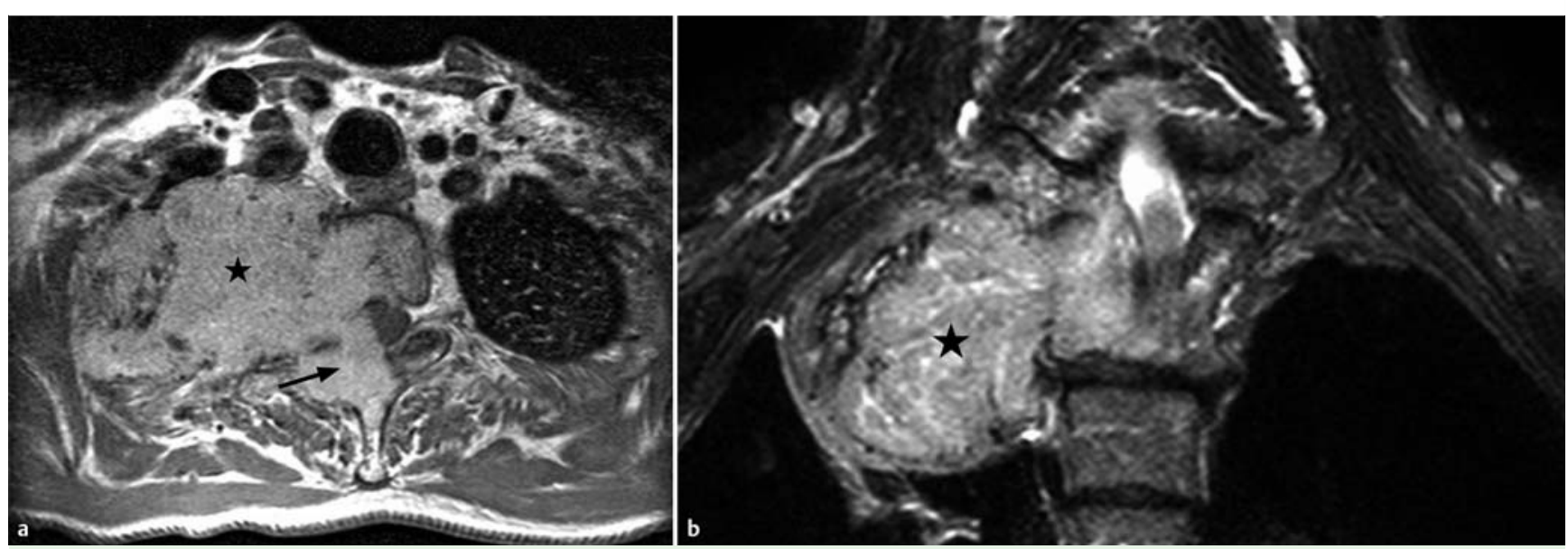

Abb. 3 Kernspintomografie HWS (axiale T2/TSE-Sequenz (a) und koronare STIR-long-TE-Sequenz [b]) 5,5 x 7,0 cm inhomogen kontrastmittelanreichernde Raumforderung $\left({ }^{*}\right)$ im Lungenapex rechts mit Infiltration der dorsolateralen Thoraxwand, Tumorwachstum per continuitatem in den HWK 7 und BWK 1 rechtsseitig mit ossärer Destruktion bis in die Processus transversii rechts (Pfeil).

(Enophthalmus, Miosis, Ptosis) kommen. Das MRT spielt bei der Diagnostik des Pancoast-Tumors eine entscheidende Rolle, da im MRT am besten das Ausmaß der Tumorinfiltration in die umgebenden Gewebe darstellbar ist. In der S3-Leitline des Bronchialkarzinoms wird bei Pancoast-Tumoren im Stadium II-IIIB eine neoadjuvante Radiochemotherapie mit anschließender Resektion empfohlen.

Ein Multiples Myelom als Ursache eines Pancoast-Tumors der Lunge ist äußerst selten. Beim Multiplen Myelom handelt es sich nach der WHO-Klassifikation um ein reifzelliges B-Zell-Lymphom, was sich als maligne Plasmazellvermehrung normalerweise auf das Knochenmark beschränkt. In seltenen Fällen kann das Multiple Myelom sich aber auch extraossär manifestieren.

\section{Interessenkonflikt}

$\nabla$

Die Autoren geben an, dass kein Interessenkonflikt besteht. 


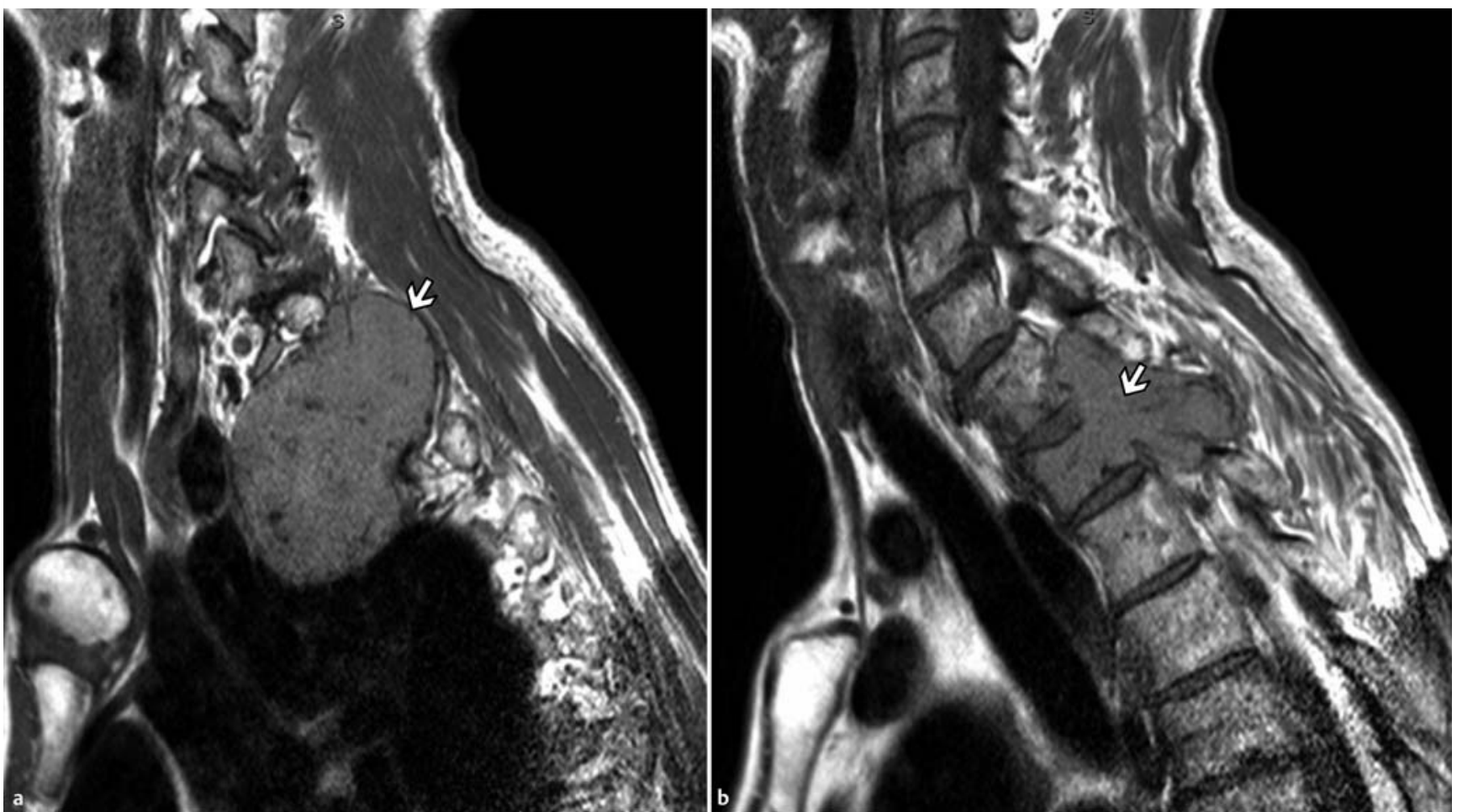

Abb.4 Kernspintomografie HWS (T1/TSE-Sequenz, sagittale Schnittebene). Kompression und Verdrängung des zervikothorakalen Myelons nach links (Pfeil) durch langstreckigen Kontakt der Raumforderung zur Dura.
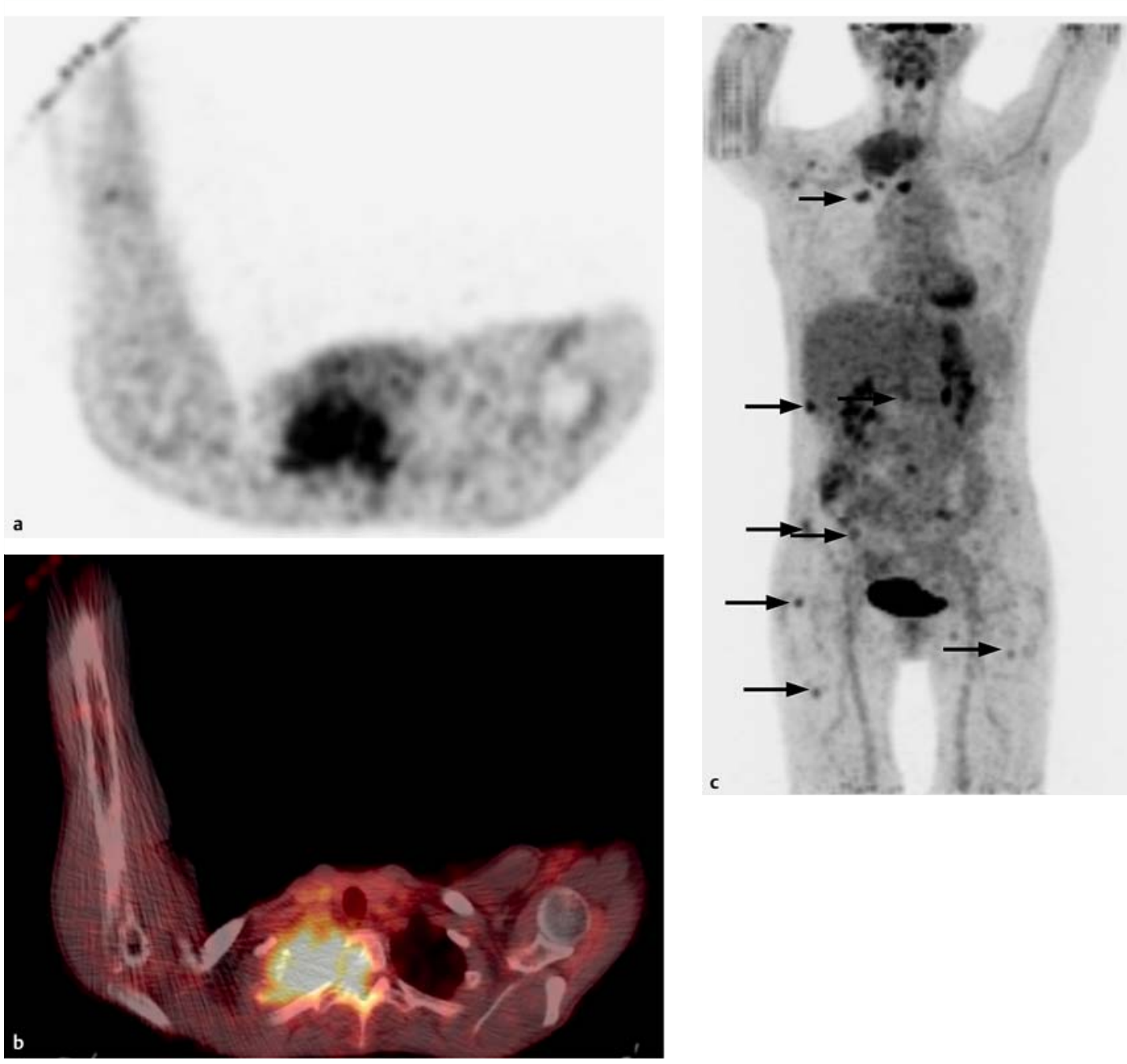

Abb.5 ${ }^{18}$ FDG PET-CT.

Ausgedehnte Tumor-

infiltration rechter Lun-

genapex mit Arrosion

der Wirbelsäule (a: PET;

b: PET-CT-Fusionsbild).

In der Maximal Intensity

Projection des PET (c)

Nachweis der multi-

lokulären Manifestatio-

nen (Pfeile) des Multi-

plen Myeloms rechter

Lungenapex sowie mul-

tipel ossär. 